\title{
Nanofibras de Algodão Obtidas sob Diferentes Condições de Hidrólise Ácida
}

\author{
Eliangela de M. Teixeira, Cauê R. de Oliveira, Luiz H. C. Mattoso \\ Embrapa Instrumentação Agropecuária, São Carlos/SP
}

\author{
Ana C. Corrêa \\ Embrapa Instrumentação Agropecuária, São Carlos/SP \\ Departamento de Engenharia de Materiais, UFSCar \\ Priscila D. Paladin \\ Embrapa Instrumentação Agropecuária, São Carlos/SP \\ Departamento de Química, UFSCar
}

\begin{abstract}
Resumo: As propriedades de termo estabilidade das nanofibras de celulose estão relacionadas diretamente com a sua aplicação e em especial ao processamento de polímeros cujas temperaturas típicas de processamento encontram-se em torno de $200{ }^{\circ} \mathrm{C}$. Neste trabalho, nanofibras de algodão comercial foram extraídas empregando-se diferentes ácidos: ácido sulfúrico, ácido clorídrico e mistura (1:1; ácido sulfúrico: ácido clorídrico). As nanofibras resultantes foram caracterizadas quanto à morfologia por microscopia de transmissão (MET), potencial zeta, análise elementar, cristalinidade por difração de raio X (DRX) e estabilidade térmica em atmosfera de ar (TGA). Os resultados indicaram uma morfologia muito similar entre as nanofibras bem como a cristalinidade das mesmas, independentemente do ácido utilizado na hidrólise. A principal diferença encontrada foi em relação ao estado de agregação das mesmas e quanto à estabilidade térmica. $\mathrm{O}$ estado de agregação das nanofibras em suspensões seguiu a ordem $\mathrm{HCl}>\mathrm{H}_{2} \mathrm{SO}_{4}: \mathrm{HCl}_{2}>\mathrm{H}_{2} \mathrm{SO}_{4}$ devido ao aumento da carga superficial nesta ordem avaliada via medidas de potencial zeta. A incorporação de $\mathrm{HCl}$ ao $\mathrm{H}_{2} \mathrm{SO}_{4}$ para a obtenção de nanofibras de celulose

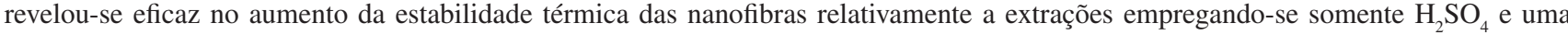
melhor dispersão em relação a nanofibras obtidas com o uso somente de $\mathrm{HCl}$.
\end{abstract}

Palavras-chave: Algodão, nanofibras de celulose, hidrólise ácida.

\section{Cotton Nanofibers Obtained by Different Hydrolytic Acid Conditions}

Abstract: The thermal stability of cellulose nanofibers is related to their application and especially to polymer processing normally occurring at ca. $200^{\circ} \mathrm{C}$. In this work, nanofibers of commercial cotton were obtained by acid hydrolysis employing different acids: sulfuric, hydrochloric and a mixture (1:1; sulfuric acid: hydrochloric acid).The morphology of the nanofibers were characterized by transmission microscopy (TEM), zeta potential, elemental analysis, crystallinity by X ray diffraction (XRD) and thermal stability (TGA) in air atmosphere by thermogravimetric analysis. The results indicated a very similar morphology and crystallinity among them. The main differences were lie in the aggregation state and thermal stability. The aggregation state of the suspensions decreases in the order $\mathrm{HCl}_{2}<\mathrm{H}_{2} \mathrm{SO}_{4}: \mathrm{HCl}_{2}<\mathrm{H}_{2} \mathrm{SO}_{4}$ due to the increase in surface charge as determined by zeta potential measurements. The hydrolysis with a mixture of $\mathrm{HCl}_{2}$ and $\mathrm{H}_{2} \mathrm{SO}_{4}$ resulted in cellulose nanofibers with higher thermal stability than those hydrolyzed with $\mathrm{H}_{2} \mathrm{SO}_{4}$. The hydrolysis employed with a mixture of sulphuric and hydrochloric acids also showed a better dispersion than those suspensions of nanofibers obtained by hydrolysis with only $\mathrm{HCl}$.

Keywords: Cotton, cellulose nanofiber, acid hydrolysis.

\section{Introdução}

Suspensões aquosas de nanofibras de celulose podem ser preparadas por hidrólise ácida gerando-se partículas coloidais de alta cristalinidade. Estas podem se apresentar com aspecto físico de finas hastes aciculares de dimensões nanométricas (diâmetro na faixa de 5 a $90 \mathrm{~nm}$ ) com alta razão de aspecto (comprimento/diâmetro). São empregados como reforço para matrizes poliméricas e os nanocompósitos resultantes geralmente apresentam propriedades superiores de estabilidade térmica, resistência mecânica e de permeação de líquidos e gases, mesmo quando as nanofibras estão em baixa concentração ${ }^{[1]}$. Entre outros pontos relevantes que estimulam o seu emprego destacam seu caráter biodegradável, baixo custo e o fato de serem obtidas a partir de fontes naturais renováveis altamente abundantes. Entretanto, a incorporação de grupos sulfatos na superfície da celulose após a hidrólise exerce efeito catalítico nas suas reações de degradação térmica. Este efeito se deve às reações de desidratação da celulose ocasionada pelo ácido sulfúrico. A água liberada catalisa estas reações por hidrólise das cadeias de celulose. Outro efeito reportado é que se tem a substituição dos grupos $-\mathrm{OH}$ da celulose, por grupos sulfatos, o que acarreta na diminuição da energia de ativação para a degradação das cadeias de celulose ${ }^{[2,3]}$. Ou seja, em geral, as nanofibras de celulose obtidas via hidrólise com ácido sulfúrico degradam-se em temperaturas menores do que sua fibra de origem. $\mathrm{O}$ uso de $\mathrm{HCl}$ para a obtenção de nanofibras de celulose já é reportado ${ }^{[2,3]}$, e, estudos revelam que o uso somente de $\mathrm{HCl}$ para a hidrólise aumenta a estabilidade térmica das nanoceluloses, mas como os íons cloretos são facilmente eliminados com repetidas lavagens com água, não há forças eletrostáticas suficientes para causar repulsão entre as partículas e consequentemente há um alto nível de formação de agregados. Neste trabalho é proposto então 
um estudo de extração e caracterização de nanofibras de algodão empregando-se uma mistura de ácidos (sulfúrico e clorídrico), investigando-se as propriedades das nanoestruturas resultantes, comparando-as com as de nanofibras extraídas com cada um dos tipos de ácidos. As expectativas foram de obtenção de nanofibras mais dispersas em relação às obtidas somente com $\mathrm{HCl}$ e de maior estabilidade térmica relativamente às nanofibras obtidas somente com $\mathrm{H}_{2} \mathrm{SO}_{4}$. Caracterizações morfológicas, cristalinidade e estabilidade térmica das nanofibras foram investigadas. Fibras de algodão constituem uma opção bastante interessante quanto ao seu uso para obtenção destas nanofibras, visto que, seu teor de celulose é superior em relação às fibras lignocelulósicas, o que evita o processo intermediário de branqueamento para remoção de outros constituintes não celulósicos ${ }^{[4]}$. Aplicações futuras de tais nanomateriais direcionam-se à obtenção de nanocompósitos empregando-os em matrizes poliméricas biodegradáveis.

\section{Experimental}

\section{Materiais}

Fibras de algodão comercial foram usadas para a obtenção de nanofibras. Os ácidos empregados foram: $\mathrm{H}_{2} \mathrm{SO}_{4}$ e $\mathrm{HCl}$ (Synth). A membrana para diálise foi oriunda da Sigma-Aldrich (D9402).

\section{Extração das nanofibras}

A extração das nanofibras de foi feita por hidrólise ácida empregando-se solução de ácido sulfúrico $\left(\mathrm{H}_{2} \mathrm{SO}_{4} 60 \%\right.$ (v/v) ou $\mathrm{HCl}\left(1 \mathrm{~mol} . \mathrm{L}^{-1}\right)$ ou $\mathrm{H}_{2} \mathrm{SO}_{4}: \mathrm{HCl}(1: 1 ; \mathrm{v} / \mathrm{v})$ a $45^{\circ} \mathrm{C}$ e 75 minutos, sob agitação vigorosa e constante. A suspensão resultante foi submetida à centrifugação e diálise em água para a remoção do excesso de ácido (pH entre 6 e 7). Em seguida a suspensão foi ultrasonificada (BRANSON 450) por 5 minutos e armazenada sob refrigeração. As condições de extração adotadas foram baseadas nos estudos específicos de Dong et al. ${ }^{[5]}$ sobre obtenção de nanofibras a partir de algodão microcristalino empregando-se ácido sulfúrico. Estas condições foram extendidas para a extração com os demais tipos de ácido ( $\mathrm{HCl}$ e mistura). A proporção entre os ácidos sulfúrico e clorídrico foi escolhida de maneira exploratória.

\section{Caracterização}

\section{Análise por microscopia eletrônica de transmissão (MET)}

Preparou-se uma solução diluída das nanofibras e, uma gota desta suspensão foi colocada sobre suportes ultrafinos ("grides") de cobre (400 mesh, Ted Pella - $\mathrm{N}^{\circ}$ 01822) e deixada secar a temperatura ambiente. Transcorridas 24 horas, as amostras foram coradas com solução 1,5\% de acetato de uranila, por imersão dos grides por 2 minutos nesta solução de contraste. As análises foram realizadas em um equipamento TecnaiTM G2 F20.

\section{Análise de potencial zeta}

A presença de cargas superficiais foi estimada via análise do potencial zeta a partir de alíquotas das suspensões aquosas das nanofibras cujas concentrações foram igualadas a $0,005 \%(\mathrm{~g} / \mathrm{g})$. Estas foram medidas utilizando-se um equipamento Malverne 3000 Zetasizer NanoZS, (Malverne Instruments, UK). Três medidas de cada suspensão foram realizadas.

\section{Análise elementar}

Análise elementar foi realizada com o objetivo de se verificar a presença de enxofre nas nanofibras após a extração. As medidas foram realizadas em um equipamento EA1110-CHNS-O da CE Instruments.

\section{Análise por difração de raio X (DRX)}

Os difratogramas de raio $\mathrm{X}$ foram obtidos a temperatura ambiente e no intervalo de ângulos $2 \theta$ variando de 5 a $40^{\circ}$. O aparelho utilizado foi um difratômetro Universal de raio $\mathrm{X}$, Carl-Zeiss-Jena, modelo URD6 a uma velocidade de $1,2{ }^{\circ} \mathrm{C} / \mathrm{min}$ operando a uma potência gerada de $40 \mathrm{kV}$ e $20 \mathrm{~mA}$ e radiação de $\mathrm{CuK} \alpha(\lambda=1,5406 \AA)$. Os índices de cristalinidade foram calculados por deconvolução dos picos dos difratogramas, tomando-se uma função de distribuição gaussiana como formato dos picos cristalinos e amorfo. Utilizando-se o software Origin 7.5, foram estimados os índices de cristalinidade com base nas áreas sob os picos cristalinos e o amorfo após a correção da linha base

\section{Análise termogravimétrica (TGA)}

A temperatura de decomposição térmica da fibra e nanofibras foram avaliadas empregando-se a técnica de termogravimetria utilizando-se um equipamento da TA Q500 (TA Instruments) nas seguintes condições: atmosfera de ar sintético; fluxo $60 \mathrm{~mL} / \mathrm{min}$; razão de aquecimento: $10{ }^{\circ} \mathrm{C} / \mathrm{min}$; intervalo de temperatura: 25 a $900{ }^{\circ} \mathrm{C}$ e porta amostra de platina. A temperatura inicial de degradação térmica $\left(\mathrm{T}_{\mathrm{id}}\right)$ ou temperatura de "onset" foi determinada através da curva TGA, como sendo a intersecção da linha de extrapolação do início do evento térmico com a tangente à curva gerada no intervalo da reação de decomposição da celulose.

\section{Resultados e Discussão}

\section{Morfologia e potencial zeta das nanofibras}

A Figura 1 mostra o aspecto físico bem como a morfologia das suspensões de nanofibras obtidas (imagens de MET). As alíquotas das suspensões aquosas foram coletadas após a sonificação e deixadas e repouso por 24 horas. É interessante observar que, imediatamente após o processo de sonificação, todas as suspensões apresentaram-se homogêneas e bem dispersas. Entretanto, com o tempo de repouso, dependendo da presença ou não de carga superficial nas nanofibras, estas se aglomeraram no caso das extraídas com $\mathrm{HCl}$ e mistura ácida. Como pode ser observado através da Figura 1, a estabilidade destas diminui na ordem $\mathrm{HCl}<\mathrm{H}_{2} \mathrm{SO}_{4}: \mathrm{HCl}<\mathrm{H}_{2} \mathrm{SO}_{4}$ indicando uma maior aglomeração das nanofibras extraídas com $\mathrm{HCl}$. Aumentando-se a proporção de ácido sulfúrico na mistura de ácidos provavelmente esta dispersão na amostra $\mathrm{H}_{2} \mathrm{SO}_{4}: \mathrm{HCl}$ seria aumentada devido ao aumento de cargas superficiais responsáveis pela repulsão eletrostática.

Exceto para a nanofibra extraída com $\mathrm{H}_{2} \mathrm{SO}_{4}$ as dimensões precisas das demais nanofibras não puderam ser determinadas com devido à alta aglomeração das mesmas. Os valores de comprimento e de diâmetro para as nanofibras extraídas com $\mathrm{H}_{2} \mathrm{SO}_{4}$ foram de $150 \pm 50 \mathrm{~nm}$ e $14 \pm 5 \mathrm{~nm}$, respectivamente, valores estes comuns a nanofibras de algodão microcristalino ${ }^{[1,5]}$. Para os demais tipos de nanofibras, pode-se observar apenas uma pequena tendência das nanofibras em apresentarem-se mais finas com diâmetros entre 9,0 $\pm 3 \mathrm{~nm}$, resultado este não tão significativo em termos de diferença em relação às extraídas com $\mathrm{H}_{2} \mathrm{SO}_{4}$. Sendo que a preparação das amostras para análises de MET seguiram as mesmas condições, nota-se o grande estado de aglomeração das nanofibras obtidas com $\mathrm{HCl}$ enquanto que as nanofibras extraídas com $\mathrm{H}_{2} \mathrm{SO}_{4}: \mathrm{HCl}$ encontram-se um pouco mais dispersas em relação as extraídas somente com $\mathrm{HCl}$. Isso pode ser justificado devido a diferenças de cargas superficiais presentes nas nanofibras. Como mostram os dados da Tabela 1, as nanofibras extraídas com $\mathrm{H}_{2} \mathrm{SO}_{4}$ apresentam maior carga superficial o que faz com que haja uma maior repulsão eletrostática tornando a suspensão mais estável em meio aquoso. As nanofibras extraídas com a mistura de ácido 


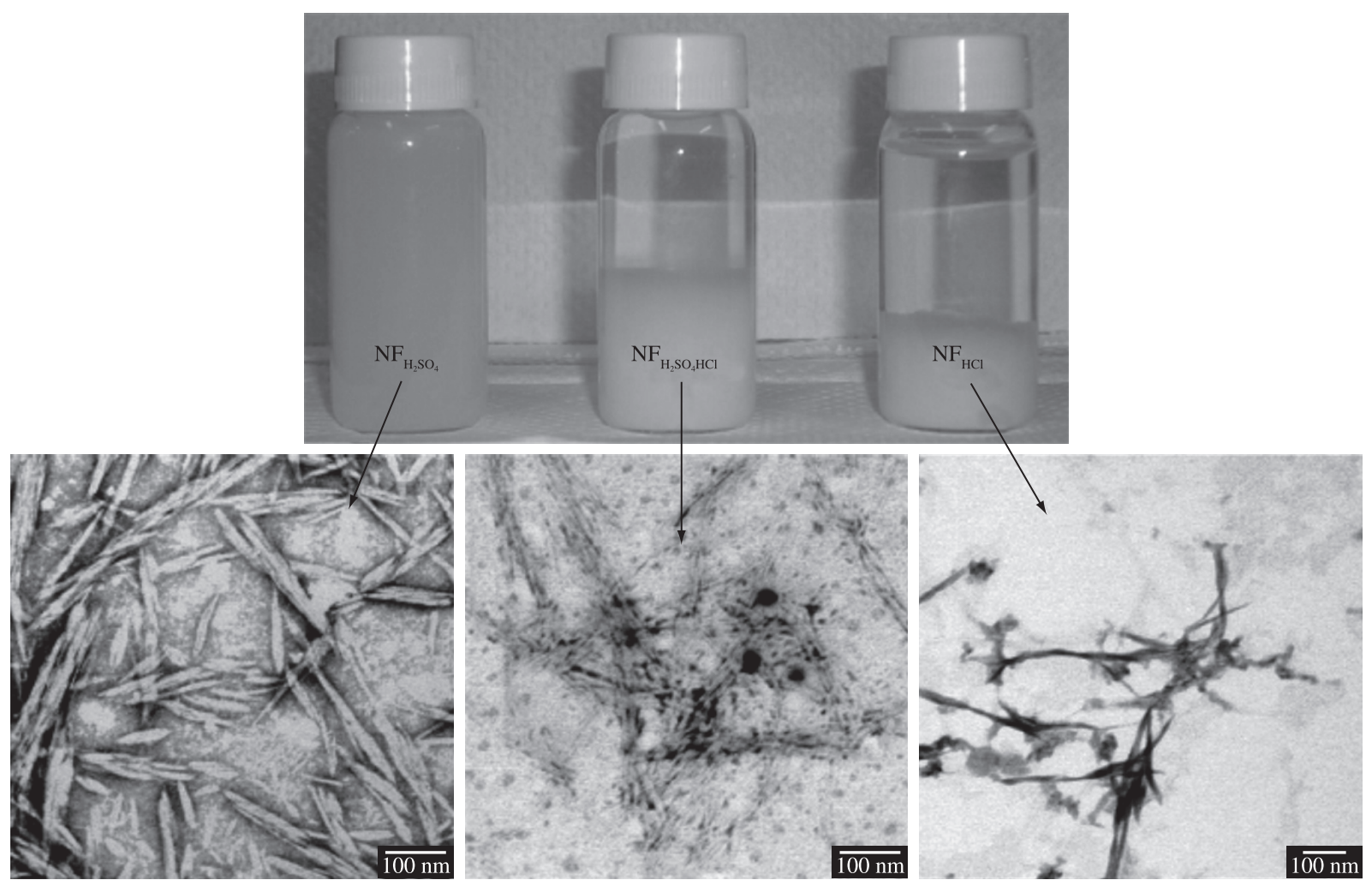

Figura 1. Suspensões de nanofibras (NF) obtidas sobre diferentes condições de hidrólise ácida conforme indicado na figura e suas respectivas imagens de microscopia eletrônica de transmissão (MET).

Tabela 1. Potencial zeta, análise elementar e índice de cristalinidade da fibra e nanofibras de algodão.

\begin{tabular}{|c|c|c|c|c|c|c|}
\hline \multirow[t]{2}{*}{ Amostra } & \multirow[t]{2}{*}{ Potencial zeta (mV) } & \multicolumn{4}{|c|}{ Análise elementar } & \multirow[t]{2}{*}{$\mathbf{I}_{\mathrm{C}}(\%)$} \\
\hline & & $\% \mathbf{N}$ & $\% \mathrm{C}$ & $\% \mathbf{H}$ & $\% \mathrm{~S}$ & \\
\hline Fibra de algodão & n. d. (*) $^{(*)}$ & 0,0711 & 41,8379 & 6,3664 & 0 & 62 \\
\hline Nanofibra $\mathrm{H}_{2} \mathrm{SO}_{4} / \mathrm{HCl}$ & $-15,7 \pm 3$ & 0,0142 & 41,6491 & 6,4870 & 1,5015 & 75 \\
\hline Nanofibra $\mathrm{HCl}$ & $-6,29 \pm 1$ & 0,0150 & 54,486 & 9,160 & 0 & 71 \\
\hline
\end{tabular}

(*) n.d - não determinado.

apresentaram valor intermediário de potencial zeta enquanto que as extraídas com somente $\mathrm{HCl}$, o valor deste foi o menor justificando a alta aglomeração das partículas. A quantidade de enxofre presente, verificada através de análise elementar (Tabela 1) é correspondente com a quantidade de carga superficial obtida através de análises de potencial zeta indicando que a presença de grupos sulfatos são os maiores responsáveis pela estabilidade das suspensões.

\section{Cristalinidade das nanofibras}

Os difratogramas de raio $\mathrm{X}$ das nanofibras são apresentados na Figura 2 onde os picos em $2 \theta \sim 15^{\circ}, 17^{\circ}, 22,6^{\circ}$ e $34,5^{\circ}$ indicam a presença de celulose tipo $\mathrm{I}^{[6]}$ tanto na fibra quanto nas nanofibras e são atribuídos aos planos (101), (1 0 - 1$)$, (002), e (040) respectivamente ${ }^{[7]}$. Para $\mathrm{NF}_{\mathrm{H}_{2} \mathrm{SO}_{4}}$ picos adicionais aparecem na região de $2 \theta \sim 11,4^{\circ}, 29,2^{\circ}$ e $30,8^{\circ}$, não puderam ser identificados

Os valores dos índices de cristalinidade das amostras encontram-se na Tabela 1.

Nota-se um aumento significativo dos índices de cristalinidade das nanofibras relativamente à fibra original indicando a efetividade das diferentes hidrólises na remoção da fase amorfa da fibra. Mas independentemente do processo de extração, o índice de cristalinidade das nanofibras obtidas foi bastante similar.

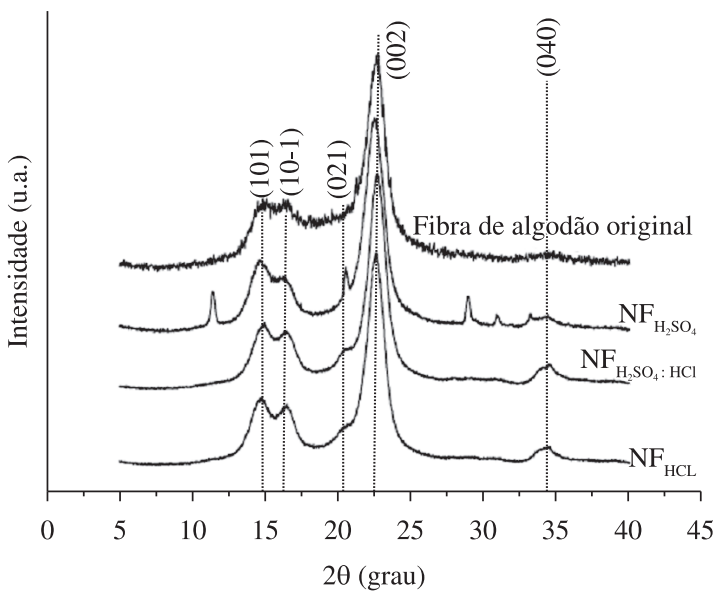

Figura 2. Difratogramas de raio X da fibra de algodão e das nanofibras.

\section{Estabilidade térmica das nanofibras}

A estabilidade térmica da fibra de algodão e das nanofibras extraídas podem ser verificadas através das curvas TGA/DTG como mostra a Figura 3. 


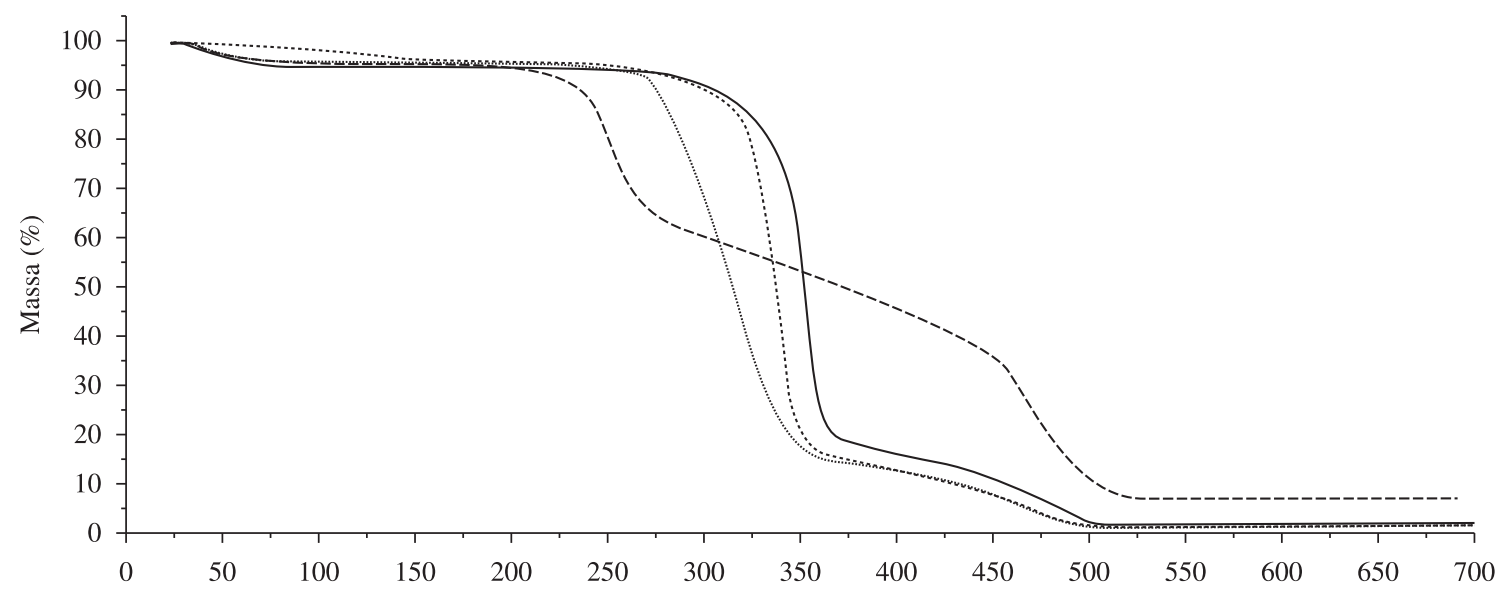

(a)

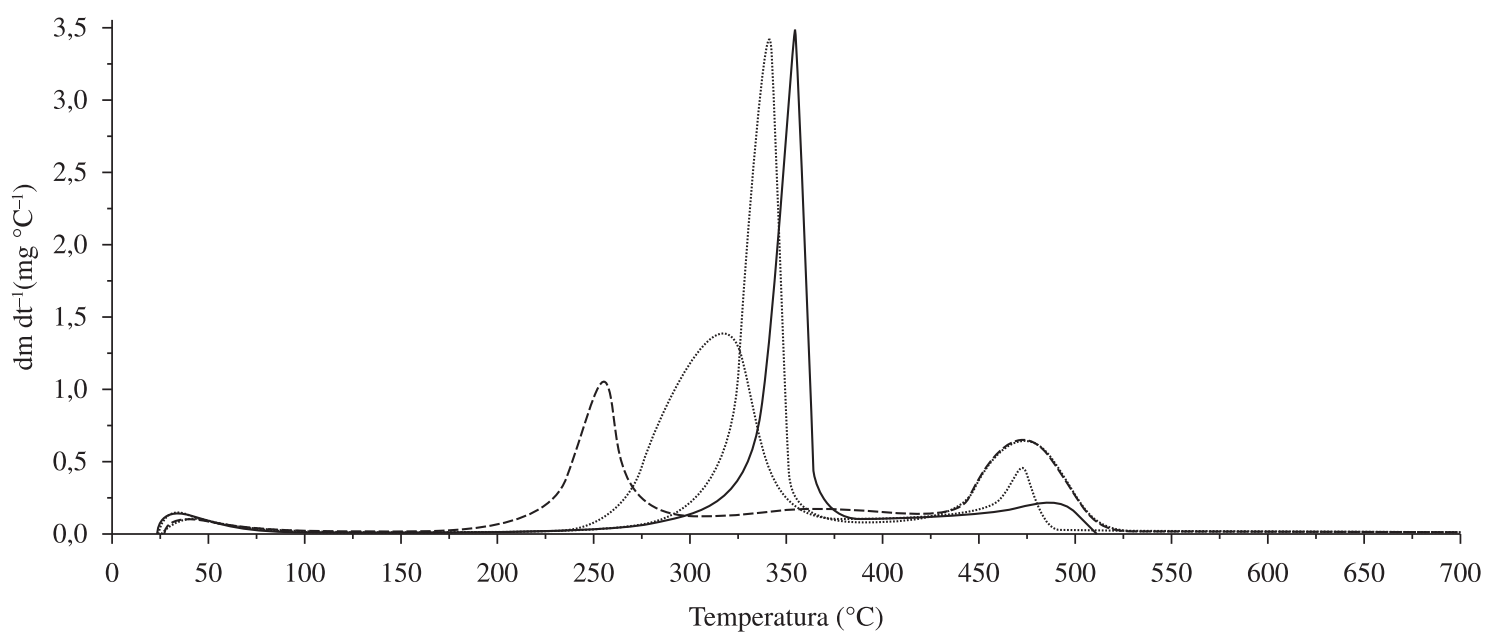

(b)

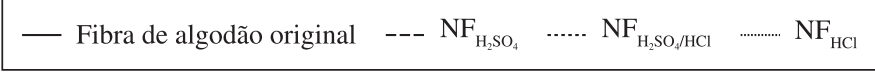

Figura 3. Curvas a) TGA e b) DTG da fibra de algodão e das nanofibras em ar sintético.

Os valores da temperatura inicial de degradação térmica $\left(\mathrm{T}_{\mathrm{id}}\right)$ e conteúdo residual de cinzas desta amostras estão reportados na Tabela 2.

Observa-se que a nanofibra obtida com $\mathrm{H}_{2} \mathrm{SO}_{4}$ degrada em temperatura inferior às das demais amostras, confirmando que a introdução dos grupos sulfatos exerce um efeito catalítico nas reações de degradação térmica das nanofibras de celulose. Notase que a degradação térmica da celulose da fibra de algodão (Figura $3 \mathrm{a}$ e b) a qual envolve reações de despolimerização, desidratação e decomposição das unidades glicosídicas no intervalo de ocorre entre $320-400{ }^{\circ} \mathrm{C}$ é manifestada como um único evento principal, refletindo em um pico na curva DTG (Figura 3b) bastante definido e estreito. $\mathrm{O}$ mesmo é válido para as nanofibras extraídas com $\mathrm{HCl}$ à exceção de uma pequena antecipação do intervalo de temperatura de ocorrência do processo termo-degradativo em relação à fibra de origem por causa da hidrólise ácida que podem estar danificando a estrutura da celulose. Para a nanofibra obtida com $\mathrm{H}_{2} \mathrm{SO}_{4}$ pode-se visualizar vários eventos entre $200-425^{\circ} \mathrm{C}$ indicando diferentes níveis de sulfatação dos nanocristais de celulose $\mathrm{e}^{[2]}$. O pico entre $250-275{ }^{\circ} \mathrm{C}$ está relacionado a cristais com maior índice de sulfatação e na região de temperatura de $275{ }^{\circ} \mathrm{C}<\mathrm{T}<400{ }^{\circ} \mathrm{C}$, envolve a degradação de celulose não sulfatada e/ou que não tiveram
Tabela 2. Temperatura inicial de degradação térmica $\left(T_{i d}\right)$ e conteúdo de cinzas a $500^{\circ} \mathrm{C}$, obtidos das curvas TGA/DTG.

\begin{tabular}{lcc}
\hline \multicolumn{1}{c}{ Amostra } & $\mathbf{T}_{\text {id }}\left({ }^{\circ} \mathbf{C}\right)$ & $\begin{array}{c}\text { Conteúdo de cinzas } \\
\mathbf{a ~ 5 0 0}{ }^{\circ} \mathbf{C}(\boldsymbol{\%})\end{array}$ \\
\hline Fibra de algodão & 320 & 0,9 \\
Nanofibra $\mathrm{H}_{2} \mathrm{SO}_{4}$ & 210 & 10,0 \\
Nanofibra $\mathrm{H}_{2} \mathrm{SO}_{4}: \mathrm{HCl}$ & 265 & 0,9 \\
Nanofibra $\mathrm{HCl}$ & 300 & 0,9 \\
\hline
\end{tabular}

contato com o ácido ${ }^{[2,8]}$. A nanofibras obtidas por hidrólise com $\mathrm{H}_{2} \mathrm{SO}_{4}: \mathrm{HCl}$ apresentou um comportamento intermediário entre as obtidas com $\mathrm{H}_{2} \mathrm{SO}_{4}$ e $\mathrm{HCl}$ manifestando um intervalo bastante largo de temperatura de degradação $\left(265-375^{\circ} \mathrm{C}\right)$ indicando a presença de nanocristais sulfatados (que se degradam em temperaturas menores) e não sulfatados.

Acima de $400{ }^{\circ} \mathrm{C}$, para todas as amostras foi manifestado um pico em torno de $400-530^{\circ} \mathrm{C}$ o qual está relacionado a degradação de resíduos carbonáceos. Verifica-se ainda através da Tabela 2 a grande quantidade de cinzas para a nanofibra $\mathrm{H}_{2} \mathrm{SO}_{4}$ em relação às demais. Segundo Roman \& Winter ${ }^{[2]}$, devido a esta característica de gerarem grande quantidade de cinzas, os grupos sulfatos podem atuar como retardantes de chama. 


\section{Conclusão}

A obtenção de nanofibras de algodão foi possível empregando-se diferentes hidrólises ácidas para a extração das mesmas. Os resultados indicaram uma morfologia muito similar entre as nanofibras bem como a cristalinidade, independentemente do ácido utilizado na extração. A principal diferença encontrada foi em relação ao estado de agregação das nanofibras em suspensão aquosa e quanto à estabilidade térmica. $\mathrm{O}$ estado de agregação das nanofibras seguiu a seguinte ordem: $\mathrm{HCl}>\mathrm{H}_{2} \mathrm{SO}_{4}: \mathrm{HCl}>\mathrm{H}_{2} \mathrm{SO}_{4}$ justificado por um aumento da carga superficial das suspensões nesta ordem verificado via medidas de potencial zeta. A incorporação de $\mathrm{HCl}$ ao $\mathrm{H}_{2} \mathrm{SO}_{4}$ para a obtenção de nanofibras de celulose revelou-se eficaz no aumento da estabilidade térmica relativamente à extrações empregando-se somente $\mathrm{H}_{2} \mathrm{SO}_{4}$ como ácido e uma melhor dispersão em relação à nanofibras obtidas com o uso de $\mathrm{HCl}$ somente. Logo nanofibras extraídas com $\mathrm{HCl}$ e mistura $\mathrm{HCl}: \mathrm{H}_{2} \mathrm{SO}_{4}$ apresentam uma boa opção para serem empregadas como reforço a matrizes poliméricas que requerem uma maior temperatura de processamento $\left(>200{ }^{\circ} \mathrm{C}\right) \mathrm{e}$ cuja incorporação se dará por processos que não envolvam meio aquoso.

\section{Agradecimentos}

Os autores agradecem à FAPESP (Processo No 07/50863-4), FINEP, CNPq e EMBRAPA.

\section{Referências Bibliográficas}

1. Dufresne, A. - J. Nanosci. Nanotechnol., 3, p.322 (2006).

2. Roman, M. \& Winter, W. T. - Biomacromol., 5, p.1671 (2004).

3. Wang, N.; Ding, E. \& Cheng, R. - Polym., 48, p.3486 (2007).

4. Mohantya, A. K.; Misra, M. \& Hinrichsen G. - Macromol. Mater. Eng., 276, p.1 (2000).

5. Dong, X. M.; Revol, J-F. \& Gray. D. G. - Cellul., 5, p.19 (1998),

6. Borysiak, S. \& Garbarczyk, J. - Fibres Text. East. Eur., 11, p. 44 (2003).

7. Sun, Y.; Lin, L.; Pang, C.; Deng, H.; Peng, H.; Li, J.; He, B. \& Liu, S. - Energy \& Fuels., 21, p.2386 (2007).

8. Li, R.; Fei, J.; Cai, Y.; Li, Y.; Feng, J. \& Yao, J. - Carbohyd. Polym., 76, p.94 (2009).

Enviado: 11/09/09

Reenviado: 20/04/10

Aceito: $26 / 04 / 10$

DOI: $10.1590 /$ S0104-14282010005000046 